\title{
A novel indoor localization scheme based on refined fingerprint-based autoencoder network
}

\author{
Junhui Mei*, and Juntong Xi \\ Shanghai Jiao Tong University, Shanghai 200240, China
}

\begin{abstract}
Indoor positioning systems have attracted increasing interests for the emergency of location based service in indoor environments. Wi-Fi fingerprint-based localization scheme has become a promising indoor localization technique due to the availability of access point (AP) and its low cost. However, the received signal strength (RSS) values are easily fluctuated by the interference of multi-path effects, which introduce propagation errors into localization results. In order to address the issue, a fingerprint-based autoencoder network scheme is proposed to learn the essential features from the measured coarse RSS values and extract the trained weight parameters of autoencoder network as refined fingerprints. The extracted fingerprints are able to represent the environmental properties and display strong robustness with fluctuated signals. The proposed scheme is further implemented in complex indoor scenes, which substantiate the effectiveness and accuracy improvement compared with other RSS-based schemes.
\end{abstract}

\section{Introduction}

With the development of location based services (LBS), localization has become one of the essential modules of wireless mobile devices. It is well known that the global positioning system (GPS) can precisely solve the localization problem for open-air environments. However, it performs ineffectively in complex indoor environments, which causes disability of GPS signals to penetrate into build-in wall materials. Therefore, a great number of researchers have been interested in proposing indoor localization schemes. Recently, a variety of wireless indoor localization techniques have been widely applied into different indoor scenes, such as Wi-Fi [1]-[4], RFID [5], Ultra Wide-Band (UWB) [6], other light signals [7], etc. As one of most attractive wireless indoor localization techniques, Wi-Fi fingerprint-based localization has been used in many emerging applications due to the availability of Wi-Fi infrastructure.

Despite these advantages, Wi-Fi fingerprint-based schemes still face challenges of improvement of localization accuracy. The localization errors come from a variety of interferences including the variability of received signal strength (RSS) in temporal and special domains, multipath effect and signal shadowing, etc. [2]. Moreover, some schemes try to incorporate human mobility captured by smartphone built-in inertial sensors to reduce localization errors [8], and some other schemes try to leverage machine learning methods to

\footnotetext{
* Corresponding author: lcchenjh@sjtu.edu.cn
} 
limit the propagation of localization errors [9]. However, the mentioned approaches above do not make substantial improvements in localization accuracy. On one side, traditional localization algorithms fail to alleviate the interference of RSS variability. On the other side, machine learning algorithms make little contribution to the reduction of localization errors at the cost of the computational complexity, which seriously occupy the computational recources of other applications on the mobile device and weaken the endurance capacity of mobile devices.

Despite these advantages, Wi-Fi fingerprint-based schemes still face challenges of improvement of localization accuracy. The localization errors come from a variety of interferences including the variability of received signal strength (RSS) in temporal and special domains, multipath effect and signal shadowing, etc. [2]. Moreover, some schemes try to incorporate human mobility captured by smartphone built-in inertial sensors to reduce localization errors [8], and some other schemes try to leverage machine learning methods to limit the propagation of localization errors [9]. However, the mentioned approaches above do not make substantial improvements in localization accuracy. On one side, traditional localization algorithms fail to alleviate the interference of RSS variability. On the other side, machine learning algorithms make little contribution to the reduction of localization errors at the cost of the computational complexity, which seriously occupy the computational recources of other applications on the mobile device and weaken the endurance capacity of mobile devices.

During the past decades, RSS fingerprint-based localization schemes have been applied into a variety of indoor environments for location based services [10]. The prevailing fingerprint-based methods mainly use the existing Wi-Fi infrastructure rather than extra deployments [11], thus resulting in low complexity of localization systems. In the previous works, several methods, including deterministic and probabilistic methodologies, were proposed for fingerprint-based indoor localization. The strategy of both two methods is searching the off-line fingerprint database and matching one or more fingerprints with the ones similar to real-time signals. Deterministic localization methods combine several Reference Points (RPs) to estimate location of the target based on the selection of the shortest distance between the real-time signal and fingerprint in multi-dimensional space [12][13]. In order to find out the shortest distance, standard norms [14] should be built for calculation. For probabilistic methods, the location estimation relies on the conditional posterior probability calculated by Bayesian rule. The statistic property of the fingerprint database is obtained from the probability distribution of the RPs data, and the property is a weighting factor for the target localization results.

In this paper, we design a fingerprint-based autoencoder network scheme for indoor localization. Our autoencoder network [15] is trained with the coarse RSS values collected at each reference point(RP) in the offline phases. In addition, the trained autoencoder network is represented by the weight parameters of each layer, which is also extracted as new fingerprints of the current RF. When the training work is conducted at each RP, the whole fingerprints extracted from trained networks construct the refined fingerprint database. In the online phase, we leverage the refined fingerprints to calculate the possibility of the target locating at RFs and estimate the target localization based on KNN [16] algorithm.

In the rest of this paper, we first provide the analysis of signals in Section 2, and then we present the architecture of the fingerprint-based autoencoder network scheme in Section 3. In Section 4, we introduce the implementation and evaluation. Finally, we conclude this paper in Section 5.

\section{Signal Analysis}


Fingerprint-based indoor localization scheme mainly has two phases: offline phase and online phase. In the offline phase, conducting site survey is responsible for collecting RSS values to construct fingerprint database and real-time RSS values are collected to estimate the target localization. The RSS values should first be established in the foundation of fingerprint-based indoor localization scheme, which is necessary during analysis of the properties of RSS values.

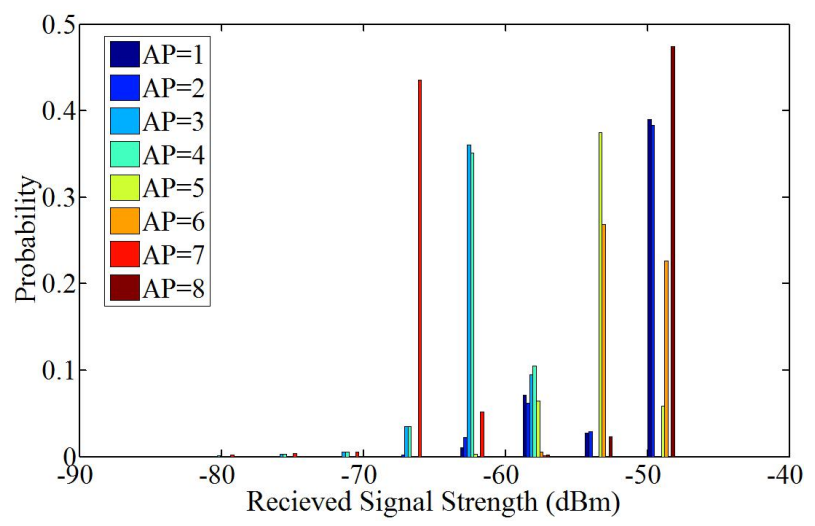

Fig. 1. CDF of correlation coefficient of RSS between adjacent positions.

Due to the complexity of indoor environment, the RSS values are susceptible to the multi-path effects, which are caused by the reflection of walls, furniture and other decorations in indoor environments. As is shown in figure 1, the RSS values collected from eight different Access Points (Aps) at the same position are variable during a short period. Thus, it is inadvisable to collect one packet of RSS vector as the fingerprint of RPs due to the variability of RSS values. Although the RSS values are inherently unstable at a single level, packets of RSS values show stable distribution. Previous works try to establish a Gaussian distribution based on the statistical property of packets of RSS data to estimate the target localization, the possibility-based method [17] improves the localization accuracy compared with traditional determine-based method. However, determine-based [18] method still faces greatly swift phenomenon in localization results due to the multi-path effects. As a result, we propose a novel autoencoder network to train refined fingerprints using the coarse RSS data. As a result, the autoencoder network shows powerful ability to extract the features from a serial of fluctuated RSS values.

\section{Method Design}

\subsection{The Architecture of Fingerprint-based Autoencoder Network}

With the availability of existing Wi-Fi infrastructure, our fingerprint-based autoencoder network scheme only leverage the mobile device to collect RSS values to localize the target without other extra deployments. As shown in figure 2, in the fingerprint training phase, the mobile device is responsible for collecting RSS values from neighboring APs as the input data of three-hidden-layer autoencoder network. The autoencoder network completes the encoding process and decoding process based on backward propagation algorithm, and then the trained weights of each layer are extracted as refined fingerprints. In the localization estimation phase, the refined fingerprints are used to calculate the possibility of the target localizing at RPs with the fusion of real-time RSS values. Based on the calculated possibility, we apply KNN algorithm to estimate the target localization. 


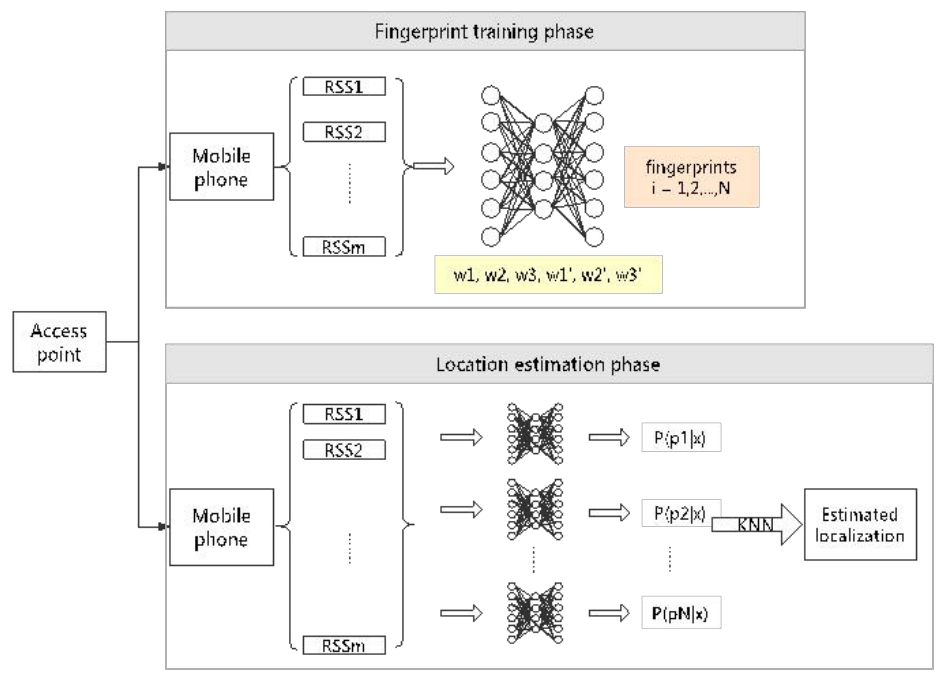

Fig. 2. The architecture of fingerprint-based autoencoder network.

\subsection{Refined Fingerprint Training in Autoencoder Network}

In the offline phase, the construction of a fingerprint database is the central step. A typical fingerprint database can be expressed as follows:

$$
\Omega=\left\{\left(\mathrm{f}_{1}, \mathrm{l}_{1}\right),\left(\mathrm{f}_{2}, \mathrm{l}_{2}\right), \ldots,\left(\mathrm{f}_{\mathrm{N}}, \mathrm{l}_{\mathrm{N}}\right)\right\}
$$

where $f_{i}$ represents the fingerprint pattern of the $i^{\text {th }} R P, l_{i}$ is the coordinate of the corresponding RP and N is the number of RPs. In the traditional method, the fingerprint pattern is the coarse RSS values and can be denoted as $\mathrm{f}=\left[\mathrm{rss}_{1}, \mathrm{rss}_{2}, \ldots, \mathrm{rss}_{\mathrm{m}}\right]$, the $\mathrm{rss}_{\mathrm{i}}$ is the measured RSS value from the $\mathrm{i}^{\text {th }} \mathrm{AP}$ and the $\mathrm{m}$ is the number of APs. However, the typical fingerprint performance is affected by signal fluctuation. As a result, we apply the coarse RSS values into an autoencoder network to train a refined fingerprint as follows:

$$
f=\left[w_{1}, w_{2}, \ldots, w_{l}, w_{1}^{\prime}, w_{2}^{\prime}, \ldots, w_{l}^{\prime}\right]
$$

where $w_{i}$ and $w_{i}^{\prime}$ denote the weights matrices of the $i^{\text {th }}$ encoding hidden layer and its decoding mirror layer, respectively. The purpose of the autoencoder network is to extract feature from the coarse RSS values. With the encoding hidden layer, it generates a target output $\mathrm{h}$ when it is fed with input $\mathrm{x}$, and $\mathrm{x}$ is a column vector of measured RSS values from detectable APs and it is same as the traditional fingerprint pattern. Then, reconstructed input $\mathrm{x}^{\prime}$ is reconstructed from the target output $\mathrm{y}$ in the decoding mirror layer. The whole learning process could be expressed as follows:

$$
\left\{\begin{array}{l}
\mathrm{h}=\phi(\mathrm{x}) \\
\mathrm{x}^{\prime}=\psi(\mathrm{h})
\end{array}\right.
$$

where $\phi$ and $\psi$ represent the encoding function and decoding function respectively. The cost function of the autoencoder network can be calculated as follows:

$$
\min _{\phi, \psi}\left\|x-x^{\prime}\right\|
$$

It is widely acknowledged that the deep learning network minimizes the cost function to obtain the optimized weights matrices, which can represent the trained deep network. By 
defining the activate function as $\varphi$, the formulas of hidden layer can be combined as follows:

$$
\left\{\begin{array}{c}
\mathrm{h}_{\mathrm{i}}=\varphi\left(\mathrm{w}_{\mathrm{i}} \mathrm{h}_{\mathrm{i}-1}+\mathrm{b}_{\mathrm{i}}\right) \\
\mathrm{h}_{\mathrm{i}}^{\prime}=\varphi\left(\mathrm{w}_{\mathrm{i}+1}^{\prime} \mathrm{z}_{\mathrm{i}+1}+\mathrm{b}_{\mathrm{i}+1}^{\prime}\right) \\
\mathcal{L}\left(\mathrm{h}_{\mathrm{i}}, \mathrm{h}_{\mathrm{i}}^{\prime}\right)=\left\|\mathrm{h}_{\mathrm{i}}-\mathrm{h}_{\mathrm{i}}^{\prime}\right\|^{2}
\end{array}\right.
$$

where $\left\{w_{i}, b_{i}\right\}$ is the weight and bias matrices of $i^{\text {th }}$ encoding hidden layer, and $\left\{w_{i}^{\prime}, b_{i}^{\prime}\right\}$ is the weight and bias matrices of $\mathrm{i}^{\text {th }}$ decoding mirror layer. The gradient descent algorithm is used to update the weights and biases matrices with the times of iteration increase until the autoencoder network obtains the optimized weights as the refined fingerprints.

\subsection{Target Location Estimation}

To estimate the target location, KNN algorithm is applied based on the data fusion. The proposed method is shown as follows:

$$
\mathrm{L}=\sum_{\mathrm{i}=1}^{\mathrm{k}} \mathrm{P}\left(\mathrm{L}_{\mathrm{i}} \mid \mathrm{x}\right) \mathrm{L}_{\mathrm{i}}
$$

where $L_{i}$ is the coordinate of $i^{\text {th }} R P$, and $P\left(L_{i} \mid x\right)$ is the posteriori probability of the target locating at $\mathrm{i}^{\text {th }} \mathrm{RP}$. According to the Bayesian law, the posteriori probability is calculated as:

$$
\mathrm{P}\left(\mathrm{L}_{\mathrm{i}} \mid \mathrm{x}\right)=\frac{\mathrm{P}\left(\mathrm{x} \mid \mathrm{L}_{\mathrm{i}}\right)}{\sum_{\mathrm{i}=1}^{\mathrm{N}} \mathrm{P}\left(\mathrm{x} \mid \mathrm{L}_{\mathrm{i}}\right)}
$$

where $P\left(L_{i}\right)$ is the prior probability and assumed to be a uniform distribution, $P\left(x \mid L_{i}\right)$ can be calculated based on the radial basis function [19] with the variance $\sigma$ of input data $\mathrm{x}$ and the refined fingerprints. $\mathrm{P}\left(\mathrm{x} \mid \mathrm{L}_{\mathrm{i}}\right)$ can be denoted in the following:

$$
\mathrm{P}\left(\mathrm{x} \mid \mathrm{L}_{\mathrm{i}}\right)=\frac{1}{\mathrm{n}} \sum_{\mathrm{i}=1}^{\mathrm{n}} \exp \left(-\frac{\left\|\mathrm{x}-\mathrm{x}^{\prime}\right\|}{\sigma}\right),
$$

where $\mathrm{n}$ denotes the number of RSS packets at each RP.

By combing with the real-time measured RSS values and the refined fingerprints, the posteriori probability is then calculated and fed to the KNN algorithm for the target location estimation.

\section{Experimental Implementation and Evaluation}

\subsection{Description of the Experiment}

Our fingerprint-based autoencoder network scheme is conducted in living complex laboratory indoor environment with many office tables and pillars that introduce several interferences into wireless signal propagation. The layout of laboratory measures $16 \times 7$ square meters with 87 training points and 46 testing points as shown in figure 3 . The left picture depicts the site where the experiments were performed, with many office tables. In the right picture, the blue circle denotes the training points fed into deep network and the red square denotes the testing points used as online test. Moreover, three lines are chosen as testing points distributed in the whole sampling points. In the fingerprint training phase, the mobile phone is used to collect coarse RSS values from neighboring APs at each RP to feed the autoencoder network. 

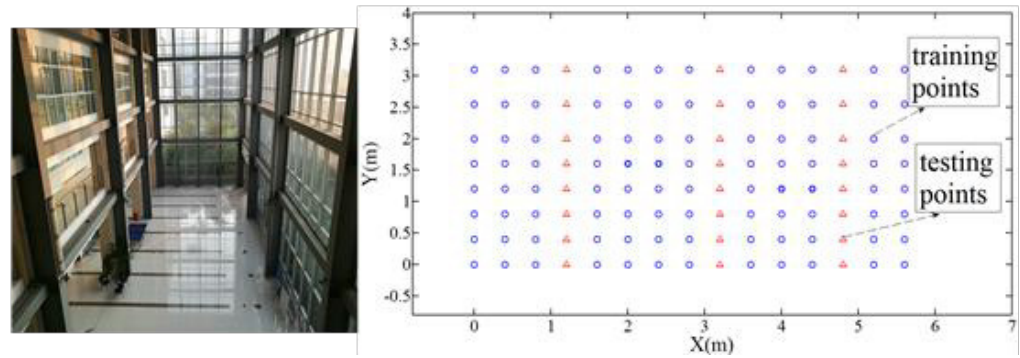

Fig. 3. Layout of the experimental lobby. (a) Real scene of lobby, (b) sampling points.

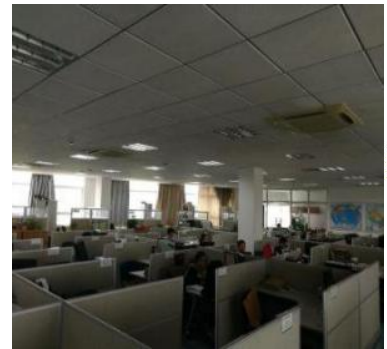

(a)

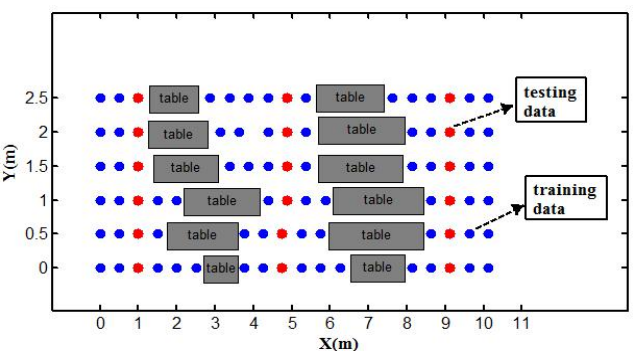

(b)

Fig. 4. Layout of the experimental laboratory. (a) Real scene of laboratory, (b) sampling points.

The parameters of our scheme mainly include the nodes numbers of three hidden layers and neighbor number $\mathrm{k}$ in $\mathrm{KNN}$ algorithm, denoting $\mathrm{n}_{1}, \mathrm{n}_{2}$ and $\mathrm{n}_{3}$ as the node number of the first hidden layer, the second hidden layer and the third hidden layer, respectively. Since the network is used to extract features, the node number of the hidden layers generally decreases along the data propagation process. Based on our collected data, the structure of autoencoder network is set as $\mathrm{n}_{1}=28, \mathrm{n}_{2}=16, \mathrm{n}_{3}=5$ and $\mathrm{k}=9$ in the $\mathrm{KNN}$ algorithm. These parameters result a good balance of localization accuracy and computational complexity.

\subsection{Experimental environments setup}

Our experiment is conducted in two phases including off-line training stage and on-line estimating stage. In the training phase, the smartphone is responsible for collecting RSS packets transformed from detected APs. In order to maintain the balance between localization accuracy and time-assumption, the number of RPs is depended on the experimental area. Once the RSS packets are collected from each RPs, the deep network can extract features from the original signal and the features are stored into off-line database as fingerprints. In the on-line estimation phase, the testing data collected by smartphone are used to estimate the target position with the match between the on-line data and off-line database.Our experiments are conducted in two typical indoor localization environments including a simple floor without obstacles and a complex laboratory with many chairs. In the first floor, in which the signals are almost no interference in transmission. As described in the figure 3, the points marked with red triangle are testing points and the points marked with blue circle are training points. Experimental area is $7 \times 4$ square meters, and hundred positions are scattered in the floor. In this area, hundreds of packets of RSS are collected from detected APs that are installed in neighboring rooms. three lines are arbitrarily chosen as testing points for validate the localization effect. The laboratory is located in the third floor, in which there are many chairs that seriously introduce much interference in the propagation of RSS. Experimental area is $12 \times 6$ square meters, as shown in figure 4, the points marked with red circle are testing points and the points marked with blue circle are 
training points. Because of the existence of barriers, only available space is selected as RPs for off-line training, which makes it more difficult to localize the target position. RSS values are collected with 0.5 grid size in continuous empty space, while it is collected with larger grid size in place where workbenches are set. Besides, the existence of pillar seriously affects the RSS propagation and make the indoor environments more complex. Due to the different size of the grid and more complex environments, the extraction of effective features are more challengeable.

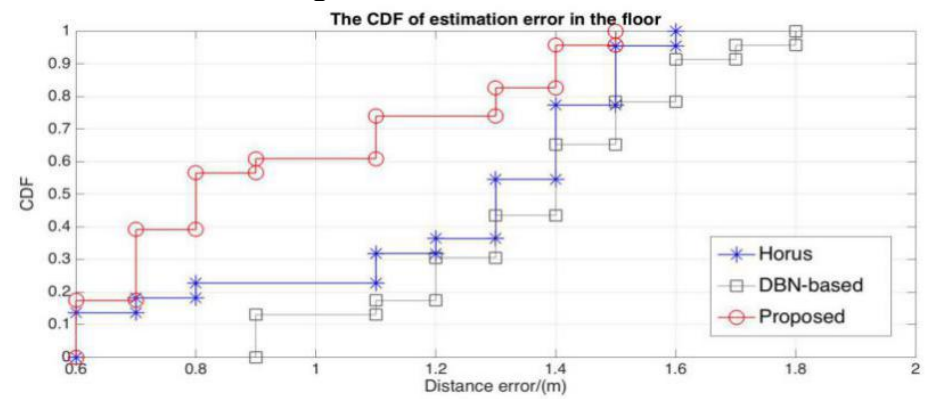

Fig. 5. CDF of estimated distance error in the floor.

\subsection{Performance Comparison}

To evaluate the performance of fingerprint-based autoencoder network scheme, we compare the performance of the proposed scheme utilizing the autoencoder network to extract features from the coarse RSS values with Horus system [20] and Zhang's method [21]. Horus system is a typical method leveraging coarse RSS values and estimating the target location in a probability-based manner. In Zhang's method, deep belief network is used to realize target location estimation, a large amount of data is needed to train the network, which takes much time to collect data and occupy much computing resource. The localization accuracy is compared in the terms of the mean localization errors $\varepsilon=$ $\sqrt{\left(x_{e}-x\right)^{2}+\left(y_{e}-y\right)^{2}}$, where $\left[x_{e}, y_{e}\right]$ and $[x, y]$ denote the estimated and the real coordinates of the test points.

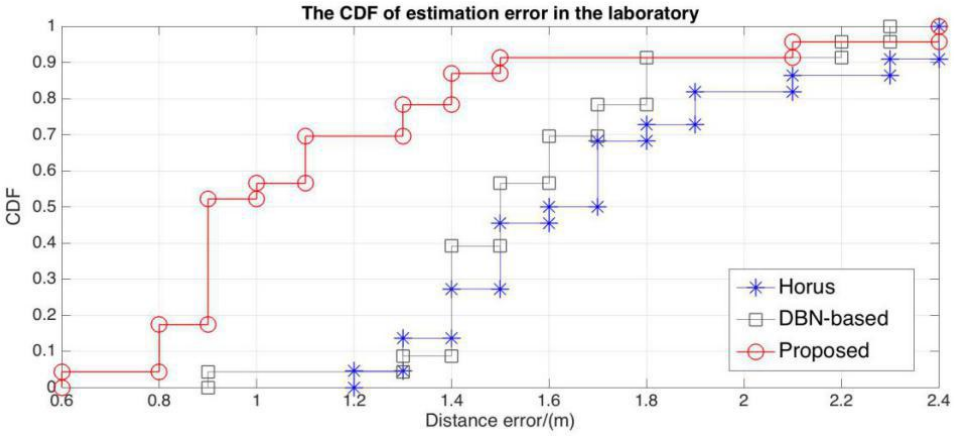

Fig. 6. CDF of estimated distance error in the laboratory.

Table 1. Localization accuracy comparison.

\begin{tabular}{|c|c|c|}
\hline \multirow{2}{*}{ method } & \multicolumn{2}{|c|}{ Mean error in two scenes (m) } \\
\cline { 2 - 3 } & floor & laboratory \\
\hline Horus [20] & 1.20 & 1.85 \\
\hline DBN-based [21] & 1.35 & 1.57 \\
\hline Proposed Scheme & 0.93 & 1.12 \\
\hline
\end{tabular}


As is shown in figure 5 and figure 6, our scheme performs better than Horus scheme and DBN-based scheme in the two different experimental scenes. In the first experiment which is conducted in floor, proposed scheme achieves 0.8 meters distance error and 1.4 meters distance error for $50 \%$ and $90 \%$ of total testing points. In addition, its mean distance error is 0.93 meter as shown in the table 1. Horus scheme has 1.3 meters and 1.5 meters of location error for $50 \%$ and $90 \%$ of total testing points, while the mean error is about 1.20 meters worse than our proposed scheme. DBN-based scheme produces 1.3 meters and 1.6 meters distance meter for $50 \%$ and $90 \%$ of total testing points, and its mean error is 1.35 meters, which is superior to Horus but inferior to our proposed scheme. In the second experiment which is conducted in laboratory, proposed scheme achieves 0.9 meters distance error and 1.4 meters distance error for $50 \%$ and $90 \%$ of total testing points as shown in figure 6 , and its mean distance error is 1.12 meters as shown in the table 1 . DBNbased scheme achives 1.5 meters and 2.1 meters of location error for $50 \%$ and $90 \%$ of total testing points, while the mean error is about 1.57 meters worse than our proposed scheme. Horus scheme produces 1.6 meters and 2.4 meters distance meter for $50 \%$ and $90 \%$ of total testing points, and its mean error is 1.85 meters, which is worse than the DBN-based and the proposed scheme. It is concluded that the proposed scheme outperforms Horus and DBN-based schemes in both environments.

\section{Conclusion}

In this paper, we present a novel fingerprint-based autoencoder network scheme. Using the coarse measured RSS values as refined fingerprints for training, weight parameters have been extracted from autoencoder network successfully, which demonstrates strong robustness capability to the variability of signals caused by multi-path effect. Compared with two other RSS-based methods, our experiment in complex laboratory indoor condition have demonstrated superior accuracy in localization performance. Further work will focus on the improvement of refined-fingerprint-based indoor localization using reinforcement learning methods.

\section{Acknowledgments}

The work described in the paper was supported by Scientific Research Project of Shanghai Science and Technology Committee (15111107902) and National Key Technology Research and Development of the Ministry of Science and Technology of China (04 project 2014ZX04015021).

\section{References}

1. He, Suining, and S-H. Gary Chan. "WiFi fingerprint-based indoor positioning: Recent advances and comparisons."IEEE Communications Surveys \& Tutorials 18(1) 466-490 (2016)

2. Davidson, Pavel and Robert, Piche. "A Survey of Selected Indoor Positioning Methods for Smartphones."IEEE Communications Surveys \& Tutorials(2016).

3. Xie, Yaqin, et al. "An improved K-nearest-neighbor indoor localization method based on spearman distance."IEEE Signal Processing Letters 23(3) 351-355(2016).

4. Xiao, Jiang, et al. "FIFS: Fine-grained indoor fingerprinting system." 21st International Conference on Computer Communications and Networks (ICCCN) (2012). 
5. Ni, L M., Liu, Y., Lau, Y C., et al. LANDMARK: indoor location sensing using active RFID, Wireless Networks. 10 (6) 701-710(2014).

6. Mucchi, L., Marcocci, P.. A new parameter for UWB indoor channel profile identification, IEEE Transactions on Wireless Communications. 8(4) 1597-1602(2009).

7. Y. -S.Kuo, P. Pannuto, K.-J. Hsiao, and P. Dutta, "Luxapose: Indoor positioning with smart phones and visible light," in Proc, ACM MobiCom, pp. 447-458.(2014).

8. Wen, Y., Tian, X., Wang, X., et al. Fundamental limits of RSS fingerprinting based indoor localization. International conference on computer communications. Pp.24792487 (2015).

9. Zhou, Rui, et al. "An Optimized Space Partitioning Technique to Support Two-Layer WiFi Fingerprinting."Wireless Communications and Networking Conference (WCNC) (2017).

10. S. Yang, P. Dessai, M. Verma, and M. Gerla, "FreeLoc: Calibration-free crowdsourced indoor localization," in Proc, IEEEINFOCOM, pp. 2481-2489 (2013).

11. C. Wu, Z. Yang, Y.Liu, and W. Xi, "WILL: Wireless indoor localization without site survey," IEEE Trans, Paralell Distrib. Syst. 24(4), 839-848 (2013).

12. Kanaris, L.; Kokkinis, A.; Raspopoulos, M.; Liotta, A.; Stavrou, S. Improving RSS fingerprint-based localization using directional antennas. In Proceedings of the The 8th European Conference on Antennas and Propagation (EuCAP), The Hague, The Netherlands, pp. 1593-1597(2014).

13. Kokkinis, A.; Raspopoulos, M.; Kanaris, L.; Liotta, A.; Stavrou, S. Map-aided fingerprint-based indoor positioning. In Proceedings of the 2013 IEEE 24th Annual International Symposium on Personal, Indoor, and Mobile Radio Communications (PIMRC), London, UK, pp. 270-274(2013).

14. Yeung, W.; Zhou, J.; Ng, J. Enhanced Fingerprint-Based Location Estimation System in Wireless LAN Environment. In Proceedings of the International Conference on Embedded and Ubiquitous Computing, pp. 273-284.(2007)

15. Liou, C. Y., Cheng, W. C., Liou, J. W., \& Liou, D. R.. Autoencoder for words. Neurocomputing 139, 84-96 (2014).

16. Mourao H A S, Oliveira H A B F D, Luiz D F. WiFi indoor positioning system using transmit power variation and kNN, IEEE, Conference on Local Computer Networks. pp.1-4.(2013).

17. Johan Chateau, Pierre Rousseau, Gregory Albiston, Beverley Cook, Stylianos Papanastasiou, Evtim Peytchev, "Implementation and evaluation of particle filtering for indoor positioning", IEEE Symposium on Computers and Communication (ISCC), pp. 1-6.(2014).

18. Samer Fayssal, "Reducing ambiguity in indoor tracking using point of interest", IEEE International Inter-Disciplinary Conference on Cognitive Methods in Situation Awareness and Decision Support (CogSIMA) .pp. 117-123 (2014).

19. Leonard, James A., and Mark A. Kramer. "Radial basis function networks for classifying process faults."IEEE Control Systems 11(3) ,31-38 (1991).

20. Youssef, M and Agrawala, A, "The Horus WLAN location determination system," in Proc. ACM MobiSys'05, Seattle, Wa, pp. 205-218 (2005).

21. Zhang, Wei, et al. "Deep neural networks for wireless localization in indoor and outdoor environments." Neurocomputing 194, 279-287 (2016). 